If correct, the result would show that interface superconductivity is consistent with a mechanism first advanced in 1973, which helps to explain low-temperature superconductivity. Proposed by US physicist John Bardeen and his colleagues, it posits that superconducting electron pairs are held together by an exciton, a particle produced by the scattering of electrons off a boundary. This does not explain all hightemperature superconductivity, but exploring it further might shed light on mechanisms that do.

Superconductivity is a field with a long history of mistaken claims and false hopes, and so it is unsurprising that Chu's result is provoking controversy. Critics say that it is difficult to measure superconductivity along narrow interfaces, let alone inside a crystal. And Johnpierre Paglione, a condensed-matter physicist at the University of Maryland in College Park, is dubious for a different reason. In 2012, he and his team reported ${ }^{3}$ a higher-than-expected superconducting temperature for regions within the same material. But in a paper posted on arXiv on 13 September ${ }^{4}$, the researchers say that what they saw is a bulk property of these regions,

\section{ALL IN THE FAMILY}

Superconducting temperatures have risen as new classes of material have been found.

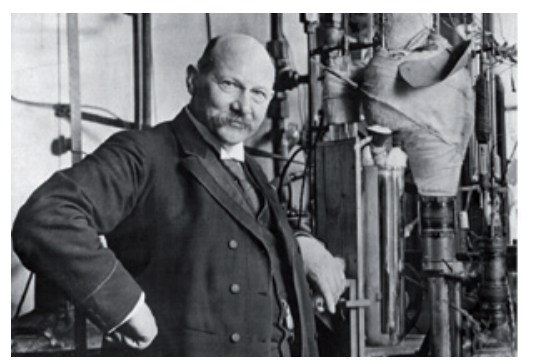

19114.2 kelvin: Mercury. Superconductivity first discovered by Heike Kamerlingh Onnes (pictured).

198630 kelvin: Barium lanthanum copper oxide. First copper-based hightemperature superconductor.

1993133 kelvin: Mercury barium calcium copper oxide. Record hightemperature superconductor.

\section{6 kelvin: Lanthanum} iron arsenide. First iron-based hightemperature superconductor.

200850 kelvin: Layered insulating and metallic copper oxide. One of the first interface-enhanced superconductors.

201349 kelvin: Calcium iron arsenide doped with rare-earth elements. Claimed single-crystal interface enhanced superconductor.

\section{AT THE INTERFACE}

Nanoscale layering in an iron-based crystal could enhance its superconductivity.

Bulk of crystal superconducts at
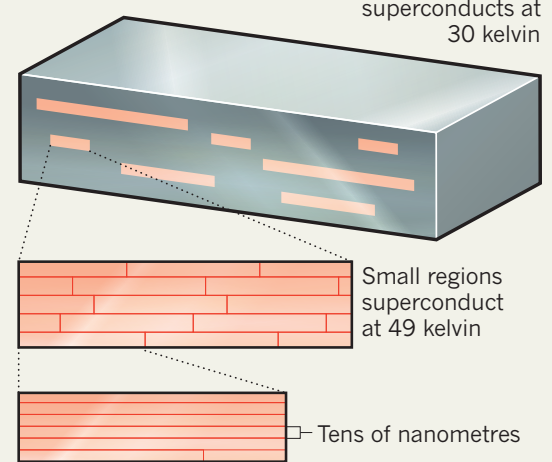

Nanoscale layers may be responsible for the enhancement

caused by the doping with rare-earth elements, rather than an interface effect.

If the superconductivity was related to interfaces, Paglione says, he would not expect it to disappear when the doping is reduced - which it does. Instead, Paglione suggests that strain in certain regions of the crystal enhances superconductivity. Chu responds that doping above a certain level helps to create interfaces by pushing arsenic atoms out of the structure.

Ivan Bozovic, a physicist at Brookhaven National Laboratory in Upton, New York, who in 2008 created one of the first interfaced superconductors, raises another possibility. $\mathrm{He}$ says that the superconductivity might not be an interface effect; instead, it could be attributable to a structural change inherent to the arsenicdepleted regions. He would like to see whether Chu can isolate these regions to study them further - and perhaps use them to create other interfaces. "We've been looking for new superconductors for 100 years and interface superconductivity is a new trick," says Bozovic, "so there's a lot of room for new combinations."

1. Wei, F. Y. et al. Preprint at http://arxiv.org/ abs/1309.0034 (2013)

2. Wang, Q.-Y. et al. Chin. Phys. Lett. 29, 037402 (2012).

3. Saha, S. R. et al. Phys. Rev. B 85, 024525 (2012).

4. Saha, S. R. et al. Preprint at http://arxiv.org/ abs/1309.3576 (2013).

\section{CORRECTIONS}

The scale on the graph showing German science spending in the News story 'Germany hits science high' (Nature 501, 289-290; 2013) had an incorrect scale. It should have run from $€ 0$ to $€ 15$ billion not $€ 0-1.5$ billion. And the News story 'Grass gets greener' (Nature 501, 291; 2013) incorrectly identified the grain that produced high yields when grown in a field that previously hosted Brachiaria grasses. It was maize (corn), not wheat. 